\title{
LA UTILIZACIÓN DE INTELIGENCIA \\ ARTIFICIAL EN LA ACTIVIDAD \\ REGULATORIA: UNA PROPUESTA EN FAVOR DEL DESARROLLO NACIONAL SOSTENIBLE
}

\author{
William Ivan Gallo Aponte ${ }^{1}$ \\ Universidad Externado de Colombia (UEXTERNADO) \\ Vivian Cristina Lima López Valle ${ }^{2}$ \\ Pontificia Universidade Católica do Paraná (PUC-PR)
}

Rafaella Natály Fácio ${ }^{3}$

Instituto de Direito Romeu Felipe Bacellar

\section{RESUMEN}

El objetivo del presente trabajo es analizar la función regulatoria a partir de la perspectiva del desarrollo nacional sostenible, entrelazando la noción de calidad regulatoria o "Smart regulation", y sus instrumentos, específicamente el Análisis de Impacto Regulatorio (AIR) realizado con el apoyo de inteligencia artificial (IA). La metodología adoptada fue deductiva, descriptiva y comparativa, conjugada con la técnica de investigación de documentación indirecta de las referencias bibliográficas y normativas. Al final, se concluye que la utilización de inteligencia artificial en el ámbito del Análisis de Impacto Regulatorio es viable y deseable para incrementar

1 Mestrando en Derecho Económico y Desarrollo en la PUC-PR. Abogado por la Universidad Externado de Colombia. Becario (bolsista) de la CAPES - Brasil. Docente investigador de la UEXTERNADO. Investigador activo del Núcleo de Investigaciones en Políticas Públicas y Desarrollo Humano (NUPED) de la PUC-PR, del grupo de investigación en Derecho del Medio Ambiente de la UEXTERNADO. Líder del grupo de investigación Serviços Públicos e Administração Pública Digital (GESPAD), de la Escuela de Derecho de la PUC-PR. Editor adjunto de la Revista Eurolatinoamericana de Derecho Administrativo. Vicepresidente de la Red Iberoamericana Juvenil de Derecho Administrativo (RIJDA). ORCID: http://orcid.org/0000-0001-7157-6291 / e-mail: williamg. aponte@gmail.com / william.gallo@uexternado.edu.co

2 Pos-doctoranda por la Universidat Rovira i Vigili (URV). Doctora y Magister en Derecho del Estado por la Universidade Federal de Paraná (UFPR). Especialista en Contratación Pública por la Universidade de Coimbra (UC). Especialista en Derecho Administrativo por el Instituto Brasileiro de Estudos Jurídicos (IBEJ). Profesora Titular de Derecho Administrativo de la PUC-PR. Coordinadora Adjunta del Curso de Derecho de la PUC-PR. Coordinadora del Curso de Especialización en Licitaciones y Contratos de la PUC-PR. Miembro del Instituto Paranaense de Direito Administrativo y del Instituto de Direito Romeu Felipe Bacellar. Miembro de la Comissão de Gestão Pública de la Ordem dos Advogados do Brasil - Seção Paraná. Abogada especializada en Derecho Público. ORCID: http://orcid.org/0000-0002-5793-2912 / e-mail. vivian.lima@pucpr.br

3 Pos-graduanda en Derecho Administrativo en el Instituto de Direito Romeu Felipe Bacellar. Bachiller en Derecho por la UFPR. Miembro fundadora de la Red Iberoamericana Juvenil de Derecho Administrativo (RIJDA) y del Instituto de Derecho Administrativo Sancionador (IDASAN). Abogada en el Escritorio Bacellar \& Andrade. ORCID: https://orcid.org/0000-0001-8580-1488 / e-mail: rafaellafacio@gmail.com

Veredas do Direito, Belo Horizonte, ·v.17 ·n.39 · p.123-146 $\cdot$ Setembro/Dezembro de 2020 
las mejoras regulatorias y contribuir para el desarrollo nacional sostenible, considerando la compatibilidad entre ambas y su aptitud para proporcionar una decisión regulatoria más técnica y efectiva. Tal práctica, entre tanto, debe ser efectiva de acuerdo con los presupuestos de dos órdenes: (1) en primer lugar, en relación a la extensión de la posibilidad de utilización de inteligencia artificial en sustitución al agente público, en ese sentido se debe alertar y hacer seguimiento a la posibilidad de que la IA sustituya la decisión del agente público; (2) en segundo lugar, con relación al modo de implementación de la inteligencia artificial, (2.1) se debe dar publicidad a la secuencia de los pasos lógicos. De la decisión algorítmica; (2.2) se debe observar las disposiciones de la Ley General de Protección de Datos, especialmente en lo que atañe al tratamiento de datos personales por el Poder Público.

Palabras clave: Análisis de Impacto Regulatorio (AIR); calidad regulatoria; desarrollo sostenible; función regulatoria; inteligencia artificial (IA).

\section{THE USE OF ARTIFICIAL INTELLIGENCE IN THE REGULATORY ACTIVITY: A PROPOSAL IN SUPPORT OF SUSTAINABLE NATIONAL DEVELOPMENT}

ABSTRACT

The objective of this work is to analyze the regulatory function from the perspective of sustainable national development, intertwining the notion of regulatory quality or "Smart regulation", and its instruments, specifically the Regulatory Impact Analysis (AIR) carried out with the support of artificial intelligence (AI). The methodology adopted was deductive, descriptive and comparative, combined with the research technique of indirect documentation of bibliographic and normative references. In the end, it is concluded that the use of artificial intelligence in the field of Regulatory Impact Analysis is viable and desirable to increase regulatory improvements and contribute to sustainable national development, considering the compatibility between the two and their ability to provide a more regulatory decision. technical and effective. Such practice, meanwhile, must be effective according to the presuppositions of two orders: (1) first, 
in relation to the extension of the possibility of using artificial intelligence as a substitute for the public agent, in that sense, it should be alerted and follow up on the possibility of AI replacing the decision of the public agent; (2) secondly, in relation to the mode of implementation of artificial intelligence, (2.1) the sequence of logical steps should be publicized. Of the algorithmic decision; (2.2) the provisions of the General Law on Data Protection must be observed, especially with regard to the processing of personal data by the Public Power.

Keywords: artificial intelligence (AI); regulatory activity; Regulatory Impact Analysis (AIR); regulatory quality; sustainable development.

\section{INTRODUCCIÓN}

En marzo del 2011, la Agencia Nacional de Vigilancia Sanitaria (ANVISA) editó una Resolución sobre la garantía de la calidad de medicamentos importados. Entre tanto, en el proceso de elaboración, revisión y aprobación de la norma, no se dejó de presente que ella contenía una disposición que generaba dudas sobre la necesidad de la manutención de muestras de referencia por parte de las importadoras. Fue justamente debido a ese tipo de ocurrencias que la referida Agencia pasó a dar atención al incremento de la calidad regulatoria, pasando a desarrollar sus primeros Análisis de Impacto Regulatorio (AIR) (ANVISA, 2018).

La regulación es objeto de múltiples transformaciones, entendimientos e incorporaciones, inclusive vanguardista en temas tecnológicos, todo con la finalidad de investigar cual es la mejor opción para tornarse más efectiva y menos costosa, tanto en términos de producción normativa, como en relación con los costos soportados por todos los actores afectados. Para atender el objetivo, queda cada vez más evidente, tanto en Brasil como en la comunidad internacional, la edición de normas no puede ser errática, es decir, apostándole entre el error y el acierto. Ella debe, sobre todo, ser precedida de metodologías específicas que lleven en cuenta todas las informaciones pertinentes, tales como la opinión de las empresas y usuarios, y también cantidades expresivas de datos que proporcionen al gestor una toma de decisión consciente a partir de un retrato fidedigno de la realidad. 


\section{EL PAPEL DE LA FUNCIÓN REGULATORIA PARA EL DESARROLLO NACIONAL SOSTENIBLE}

La construcción del Derecho Administrativo en el contexto decimonónico francés está sentada sobre el paradigma del positivismo jurídico, según el cual, la actuación de la Administración Pública tenía como único fundamento la ley. Al final de cuentas, la voluntad del soberano fue sustituida por la voluntad general, cuya expresión máxima, en los términos del dogma de Rousseau, era la ley. El espacio de la selección o escogencia administrativa era, por tanto, bastante reducida en la medida en que estaba vinculada a la subsunción a los preceptos legales estrictos (GUERRA, 2018, p. 60). Ese arquetipo de decisión comienza a modificarse con la creación del Consejo de Estado francés y con el fortalecimiento de la máquina administrativa sobre el comando de Napoleón (GUERRA, 2018, p. 64), pero es en la postmodernidad ${ }^{4}$ en donde se demuestra inequívocamente insuficiente.

El positivismo jurídico intentó desvincularse de la axiología y del contenido sustantivo en la aplicación de la ley con la pretensión de tornarse una ciencia "pura"; entre tanto, al prescindir de tales valores o presupuestos, acabó por fundamentar los más catastróficos errores históricos de abuso del ser humano. Siendo urgente, entonces, la necesidad de refundar el Derecho sobre bases distintas, que expresan valores, finalidades e intereses capitales indisponibles de la naturaleza humana. Así se culminó en el neoconstitucionalismo, modelo caracterizado por Constituciones con un extenso catálogo de Derechos Fundamentales, bien como valores y principios que desplazan la noción de legalidad para la noción de juridicidad (CARBONELL, 2003). ${ }^{5}$

La ley fundamental de 1988, como Constitución postmoderna que es, refleja esa transformación: reclama un Derecho Administrativo según el cual el Estado administra los intereses públicos que constitucionalmente le fueron atribuidos más allá de la observancia de las reglas legales emanadas de los órganos legitimados (MOREIRA, 2011, p. 19-20). Reclama,

4 El término postmodernidad fue popularizado por Jean-François Lyotard para referirse a las nuevas concepciones que surgieron después de la Segunda Guerra Mundial, así como las transformaciones posteriores en los años 80 y 90 del siglo XX. En: (LYOTARD, 1985) (GUERRA, 2018, p. 116).

5 Uno de los autores que más abordó el tema fue Miguel Carbonell. Según el autor, este fenómeno llamado neoconstitucionalismo puede analizarse en tres niveles: (i) Textos constitucionales, que hoy son más sustantivos, más valores, más derechos fundamentales, más principios; (ii) Forma de interpretación y aplicación de las normas constitucionales, considerando el uso de métodos más abiertos que el silogismo y la subsunción; (iii) Nuevos modelos teóricos para comprender la Constitución y la ley en su conjunto. 
finalmente, un Derecho Administrativo postmoderno (MOREIRA, 2008). Por tal razón, importa estudiar los contornos de ese Derecho Administrativo postmoderno específicamente con relación a la regulación independiente, pues está tiene contornos especiales en relación con los demás actos administrativos practicados en el ejercicio de la función administrativa. Esto porque las leyes que instituyeron el sistema de regulación tienen menor densidad normativa, lo que necesariamente abre un espacio para mayor discrecionalidad de la Administración Pública. Y, más allá de eso, este factor aumenta la complejidad de análisis de legalidad de los actos, teniendo en cuenta que la ausencia de una subsunción simétrica a la ley, demanda evaluaciones más complejas sobre la proporcionalidad, razonabilidad e imparcialidad (MOREIRA, 2008, p. 9).

El modelo de Estado regulador fue confirmado en Brasil con la promulgación de la Constitución de 1988 (GUERRA, 2018, p. 139). Es oportuno esclarecer que al reafirmar el modelo regulador no se pretende conciliar con la indefendible idea de que el Estado puede prescindir de la prestación de los servicios públicos que le fueron otorgados, sustituyendolos por la mera regulación, pues tal entendimiento no tiene respaldo constitucional. ${ }^{6}$ Conforme lo enuncia el artículo 175 de la Constitución, al Poder Público le incumbe prestar, y no apenas "garantizar" o "regular" la prestación del servicio público. Frente a ese entendimiento, Odete Medauar afirma que por más que la noción de servicio público sea repensada y hasta mismo con la finalidad de "insertar el dato económico, la concurrencia, la gestión privada", nunca podrá ser suprimida la presencia del Estado (MEDAUAR, 2003, p. 126).

La regulación estatal puede ser comprendida en sentido amplio o estricto. En el amplio, equivale a cualquier postura que el Estado adopte orientando hacia el correcto funcionamiento de determinado mercado, incluyendo hasta mismo la monopolización de una actividad. En sentido estricto, la regulación equivale a una acción estatal específica por medio de la cual el Estado actúa como normalizador y controlador del ejercicio de una actividad económica, cuyo objetivo también es garantizar el mejor funcionamiento y desarrollo posible del mercado regulado. Y el buen

6 Algunos autores opinan lo contrario, argumentando que las competencias de prestación de servicios públicos solo pueden ejercerse "garantizando" que la actividad se proporciona de alguna manera: (SCHIRATO, 2012, p. 138-139); o que esta actividad sea "de algún modo asegurada por el Estado": (MOREIRA NETO, 2000, p. 140-141). Sin embargo, el contenido legal de la propiedad estatal de los servicios públicos no incluye entendimientos según los cuales el Estado desempeña un papel de garante, ya sea a través de la actividad reguladora o por cualquier otro medio. En ese sentido (MELLO, 2017) y (SCHIER, 2016). 
funcionamiento del mercado no está relacionado apenas con los intereses empresariales, pero también de los usuarios de los servicios y del propio Estado (SCHIRATO, 2013, p. 4-5).

Esto es, la finalidad de garantizar el desarrollo está inmersa en el propio concepto de regulación. Pero ¿Qué sería ese desarrollo sostenible? Se trata de un vocablo polisémico y sin conceptualización legal para lo cual ya fueron atribuidas numerosos significados. Hasta la década de 1960 predominó una visión estructuralmente económica de desarrollo, entre tanto los preceptos de la Constitución brasilera de 1988 imprimió al término una facción íntimamente vinculada con el valor de la igualdad (HACHEM, 2013, p. 150). Para más allá del crecimiento económico, la noción representa un proceso de elevación continua de la vida de los ciudadanos como incremento también social y político (GABARDO, 2009, p. 243). Además, el desarrollo debe contemplar la generación presente y también las generaciones futuras, por tanto debe ser también sostenible (SCHIER, 2019, p. 42-43).

En este sentido, mucho antes de que la función regulatoria haya ganado prominencia en el contexto de la reforma administrativa de la década de los 90, cuyo cuño ideológico en relación al desarrollo era notoriamente neoliberal, ${ }^{7}$ el abanico dogmático sobre ella debe estar sentado sobre aspectos diversos, más allá del económico, enlistados encima: social y político. Nada más natural que eso, una vez que la función regulatoria del Estado, los intereses empresariales de los agentes privados y también los intereses de los usuarios de los servicios (SCHIRATO, 2013, p. 2-3).

La imbricación entre la regulación, el desarrollo y la sostenibilidad fue muy bien observada por Juarez Freitas, para quien la finalidad de la función regulatoria es defender a largo plazo la preponderancia de los principios, objetivos, y derechos fundamentales, teniendo en cuenta el "bienestar multidimensional en el presente, sin comprometer el bienestar en el futuro" (FREITAS, 2011, p. 278-279). Para el autor, el siglo XXI reclama una nueva teoría administrativa de regulación estatal como parte de una agenda de sostenibilidad, y ella debe estar basada entre otras cosas, en la buena regulación (FREITAS, 2011, p. 278-254). Al final de cuentas, la sostenibilidad exige un abanico regulatorio consistente a largo plazo (FREITAS, 2011, p. 255).

7 La observación es hecha por Daniel Wunder Hachem, quien señala como ejemplo nacional a Luiz Carlos Bresser-Pereira, uno de los principales ejes de la reforma neoliberal del Estado brasileño en la década de 1990 y también uno de los grandes autores sobre el tema del desarrollo: (HACHEM, 2013, p. 150). 
Así, conforme lo indica Vitor Rhein Schirato (2013, p.4), el desempeño de esta función de forma equivocada causa inestabilidad en las relaciones entre las partes afectadas y perjudica el desarrollo del sector regulado, lo que imposibilidad acciones sostenibles de largo plazo. La falta de exención e influencia política, por ejemplo, implica una toma de decisión no exenta y sin tecnicidad, porque el elemento primordialmente orientado no es la calidad o la sostenibilidad a largo plazo de la actividad sectorial regulada.

No obstante la importancia de la regulación para el desarrollo sostenible, el actual sistema regulatorio brasilero enfrenta una franca crisis. Para Vitor Rhein Schirato hay al menos tres presupuestos básicos que garantizan su buen funcionamiento: (i) la independencia de las autoridades reguladoras; (ii) el proceso decisorio de esas autoridades, que debe ser técnico e permeable al diálogo con los demás agentes del sector regulado, y (iii) el respeto por los órganos de control (interno y externo), de las decisiones técnicas emitidas por las autoridades reguladoras (SCHIRATO, 2013, p.12). $\mathrm{Y}$ en todos están comprometidos: (i) la independencia de las autoridades reguladoras es cada vez más mitigada por la influencia política; (ii) el proceso decisorio de las agencias es amenazado en razón de la falta de tecnicidad de sus dirigentes y del irrespeto a las normas del proceso; y (iii) los órganos de control se inmiscuyen, con frecuencia, en el campo de la discrecionalidad que es reservado a las agencias (SCHIRATO, 2013, p. 2).

En ese contexto, es imprescindible una transformación de paradigma en la forma de acción de la Administración Pública reguladora que contemple "un proceso mucho más complejo de identificación y ponderación de todos los intereses públicos subyacentes a la decisión, con vista a la emisión de una manifestación final equilibrada para el funcionamiento adecuado del sector regulado" (SCHIRATO, 2013, p. 9). El presente artículo, al proponer la incorporación del Análisis de Impacto Regulatorio con Inteligencia Artificial, como apoyo de la decisión regulatoria, pretende colaborar para la realización del presupuesto de tecnicidad de su contenido.

\section{2 "SMART REGULATION" O REGULACIÓN INTELIGENTE: CONCEPTO Y SUS TRANSFORMACIONES, INSTRUMENTOS Y EXPERIENCIA BRASILERA E INTERNACIONAL}

El concepto de smart regulation o regulación inteligente ha tenido una comprensión indiscriminada. En la mayoría de las veces, de acuerdo con 
el significado literal de sus palabras, se entiende como la acción o el efecto de regular u ordenar determinada actividad o servicio con cierta destreza, habilidad o experiencia. En esta línea general, se puede comprender la noción como un mecanismo capaz de conjugar, con cierto protagonismo, dinamismo y determinada coordinación (SILVA, 2004, p. 343).

La regulación inteligente se relaciona también con el concepto de efectividad, pues con el aumento sobre el conocimiento de procesos, funciones y estructuras reales de la economía, la educación, salud, entre otros, promueve el respeto de las peculiaridades de cada uno de los subsistemas sociales. Partiendo de esa premisa, la solución para determinado problema relacionado a la adecuación de la regulación de cierta área será concebida de forma "más inteligente" (ARAGÃO, 2000, p. 276).

Para comprender mejor la importancia de la noción de calidad regulatoria (MORENO \& GALLO, 2019) y como ella puede ser efectiva en los ordenamientos jurídicos, en especial en el brasilero, es especial delinear dos análisis: (i) una visión histórica y teórica de la noción, destacando cómo se dio su surgimiento y posteriores transformaciones hasta su actual concepción; (ii) y otra específicamente sobre uno de sus instrumentos para hacerlo efectivo, el Análisis de Impacto Regulatorio (AIR), destacando la experiencia pátria y demás países en su implementación.

El concepto de regulación inteligente fue introducido en 1998 por Neil Gunningham (2017, p. 133-149) como una propuesta alternativa de entendimiento de la regulación, a través de la cual se acoge de manera flexible, creativa e innovadora las diferentes formas de control social. De este modo, la aplicación de la regulación inteligente permitiría la participación de diversos actores que, conforme se destacó en la parte anterior, están legítimamente interesados en el proceso de regulación y deben tener su participación asegurada. Con la abertura de la participación se tiene el resultado de medidas más efectivas y eficientes que las formas tradicionales de regulación en donde apenas el Estado participa y decide.

En el ámbito de la interacción entre los diversos actores citados anteriormente, conforme lo señala Gunningham, la concepción tradicional del proceso regulatorio en el cual el Estado actúa como regulador y las empresas como terceiros "regulados" sufre una transformación sustancial. La tendencia pasa a ser la incorporación de esos interesados mediante múltiples mecanismos que permiten integrar de forma eficiente patrones o parametros, en la mayoría de los casos internacionales, com el objetivo de obtener resultados de calidad (GUNNINGHAM \& SINCLAR, 2017, p. 134).

Esa tendencia fue propuesta para analizar la falta de efectividad de 
las soluciones concebidas para los complejos problemas ambientales que el mundo sufre. Entre tanto, tomó proporciones de carácter general, en el sentido de que debe ser incorporada en los diversos segmentos regulados, razón por la cual se llega afirmar que es una nueva forma de política pública (TOFFELSON, 2008), cuyo objetivo es primordialmente responder de modo eficaz a las necesidades de los ciudadanos. ${ }^{8}$

Para Michael Moran, a diferencia de lo propuesto por Gunningham, la regulación inteligente surgió para responder a una pregunta crítica relacionada con la transición de estrategias del gobierno de la forma más sutil posible (MORAN, 2002, p. 391-413). Esta concepción, por tanto, nuevamente amplía la noción de regulación inteligente. En un primer momento, como se vio, la idea era inherente a las materias ambientales, y pasó a adoptarse en diversos ramos, todo con vista a garantizar la participación de los legítimamente interesados, en un segundo momento el objetivo no es apenas garantizar la participación de los demás actores, pero sobre todo para garantizar efectividad de la regulación.

Es precisamente esta la connotación de la regulación inteligente que se adopta en el presente trabajo, según la cual debe haber calidad regulatoria en todos los sectores regulados, cuya finalidad no es apenas el incremento de la participación de los interesados, pero también el aumento de la tecnicidad de las decisiones. Al final de cuentas, a pesar de la imprecisión del término calidad regulatoria, se puede afirmar que la noción es construida a partir de otros diversos principios más allá de la participación social, tales como: la preferencia por la variedad de instrumentos regulatorios, la opción por medidas alternativas a la regulación en exceso (MASSIMINO, 2015, p. 39) (y no desregulación) y, finalmente, la búsqueda por la maximización de resultados satisfactorios (GUNNINGHAM \& SINCLAR, 2017, p. 134).

En ese mismo sentido, la Organización para la Cooperación y Desarrollo Económico (OCDE) concibe la regulación inteligente como un instrumento para disminuir la multiplicidad de fuentes de regulación incidiendo sobre el mismo caso, lo que causa inseguridad jurídica en la medida en que los destinatarios de la norma no pueden tener certeza sobre cuál regla debe ser acatada y obedecida (KLINGBEIL, 2019) (CONSEJO EUROPEO, 2018).

Los instrumentos de mejora regulatoria fueron desarrollados a partir

8 Es el caso de Colombia, donde el concepto fue incorporado como política pública. (COLOMBIA. Departamento Nacional De Planeación (DNP), 2018). 
de las órdenes ejecutivas ("executive orders") en los Estados Unidos (BETANCOR, 2009, p. 107). La práctica fue iniciada en el referido país sobre el comando del entonces presidente Ronald Reagan, que editó órdenes determinado que la regulación fuese obligatoriamente precedida de un análisis de costo-beneficio. Había cierto escepticismo con relación a la propuesta, pues se tenía que el análisis meramente económico no fuese compatible con la regulación de temas como medio ambiente y salud. Mismo así, los presidentes Bill Clinton y Barack Obama dieron continuidad a la práctica de forma satisfactoria, pues se observó que la metodología ayudó a identificar regulaciones que no cumplían su propósito y acoger otras buenas ideas en términos de efectividad (SUSTEIN, 2019).

Esos instrumentos inicialmente concebidos en los Estados Unidos fueron reconocidos como buenas prácticas por la OCDE en 1995, y desde entonces la entidad internacional pasó a recomendar su adopción para los demás países integrantes. Estos consisten, básicamente, en una agenda regulatoria, el análisis de impacto normativo o regulatorio, la evaluación ex ante o ex post, la abogacía de la competencia, la expedición de normas y la consulta pública. El objetivo del presente trabajo será el Análisis de Impacto Regulatorio (AIR). Por tanto, se ahondará sobre la utilización de la herramienta en Brasil, no obstante, comparando la experiencia de otros países.

La actividad regulatoria ganó preeminencia en Brasil con la reforma administrativa de la década de 1990 y la creciente delegación de servicios públicos. La ideología propuesta por el entonces gobierno estaba enfocada en la "disminución del tamaño del Estado". En ese contexto, las agencias reguladoras fueron concebidas (o fue esta, por lo menos, la intención) como instituciones autónomas e independientes, cuyo objetivo era mejorar el abanico regulatorio para la obtención de resultados más efectivos (RAGAZZO, 2018). Esa necesidad inaugura "una nueva fase del debate de mejora regulatoria en Brasil" (RAGAZZO, 2018, p. 516), constituyendo un paradigma de implementación de herramientas con tendencia de sistematización y coordinación para el incremento de la calidad regulatoria.

En este contexto, ya en 2007, por el Decreto No. 6062 fue creado el Programa de Fortalecimiento de la Capacidad Institucional para la Gestión en Regulación (PRO-REG). En términos de iniciativas concretas, es relevante citar que diversas agencias (Agencia Nacional de Transportes Terrestres - ANTT, Agencia Nacional de Salud Suplementar y Agencia Nacional de Aguas) pasaron a realizar (respectivamente en 2009, 2010 y 
2015) todavía que puntualmente y de forma no obligatoria, Análisis de Impacto Regulatorio (AIR) (BRASIL, 2018).

El Análisis de Impacto Regulatorio (AIR), conforme la nomenclatura de su nombre ya anuncia, consiste en un proceso ${ }^{9}$ de análisis de los impactos de la norma regulatoria, y puede ser hecho antes de su promulgación o después, considerando que la decisión regulatoria debe ser continuamente evaluada. El propósito de la herramienta es sobretodo, proporcionar la medida del costo-beneficio de la norma a ser editada. El análisis, entre tanto, no es apenas económico, pues atañe también los aspectos sociales, político, ambiental y cultural (FERREIRA, 2015).

La utilización de esta herramienta de gestión pública para la toma de las decisiones promueve una verdadera transformación de la cultura administrativa (CASTRO, 2014). Más allá del incremento en relación a su finalidad intrínseca, que es el aumento de la efectividad de la regulación (MENENGUIN \&BIJOS, 2016, p. 7), la mejora regulatoria también se verifica en los siguientes aspectos: (i) en el aumento de la participación de los interesados, considerando la procesalización del emprendimiento; (ii) en el aumento de la tecnicidad de la decisión, delimitando de forma clara la discrecionalidad (BLANCHET \& BUBNIAK, 2017, p. 1-15), evitándose así, el uso político de la función sobre el argumento o posibilidad de la conveniencia y la oportunidad; (iii) y en el establecimiento de patrones más claros para el control del proceso decisorio (JORDÃO, et al. 2019, p. 75). ${ }^{10}$

Del punto de vista de la concreción normativa, la implementación del AIR como herramienta obligatoria es reciente. En 2015 fue presentado un proyecto de ley en el cual se establecieron directrices generales para la realización de AIR con relación a la toma de decisiones regulatorias, entre tanto, la norma jamás fue sancionada (BRASIL, 2015). En noviembre de 2017, el Gobierno Federal publicó el Decreto No. 9.203, en el cual se estableció que la aplicación de buenas prácticas regulatorias, entre ellas el AIR, como una directriz y principio de gobernanza pública. En 2018, el Gobierno Federal publicó las Directrices generales y una guía orientadora para la elaboración de AIR (BRASIL, 2018), un documento de referencia

9 Se opta por la palabra "proceso" en lugar del "procedimiento" para enfatizar que la participación de las personas interesadas y afectadas es esencial para el instituto, dirigido no solo a la efectividad de la regulación, sino también a los derechos y garantías fundamentales de los ciudadanos: (BACELLAR FILHO, 2014, p. 107-135) Para una lectura profunda sobre la procesualización de la función administrativa: (MEDAUAR, 2008).

10 En ese sentido también el Proyecto de ley n. 1.539 de 2015 da Câmara dos Deputados, que estabelece la obligatoriedad de realización de Análisis de Impacto Regulatorio en el ámbito de la Administración Federal. 
en el cual se desarrollan importantes aspectos de implementación, análisis y proyección de la herramienta de la mejora regulatoria.

En junio de 2019 fue promulgada la Ley No. 13.848, que determina la realización de AIR para la adopción y las propuestas de alteración de actos normativos de interés general de los agentes económicos, consumidores o usuarios de los servicios prestados. La interpretación de la herramienta, entretanto, depende de la promulgación de la reglamentación específica. Otra norma en la que se abordó el tema fue la llamada "MP de la libertad económica”, Medida Provisória n. 881/2019, hoy convertida en la Ley 13.874 de 2019, por medio de la cual se instituye la declaración de derechos de libertad económica, estableciendo garantías de libre mercado. La referida norma también exige AIR en casos de expedición o alteración de actos normativos de interés general de agentes económicos o de usuarios de los servicios públicos prestados (RODRÍGUEZ-ARANA, 2014), y extiende la existencia a cualquier órgano o entidad de la Administración Pública Federal, incluídas las autarquias y las fundaciones públicas.

Considerando las iniciativas y normas citadas anteriormente, es evidente que la progresiva inclusión del AIR como herramienta de mejora regulatoria dentro de la Administración Pública es un asunto contemporáneo y cada vez más frecuente. De este modo, Brasil se alinea a una tendencia internacional, considerando las ya diversas experiencias de los demás países en el tema, que serán puntualmente mencionadas a continuación. ${ }^{11}$

En México, la denominada Ley General de Mejora Regulatoria, promulgada en 2018, incluye el AIR como una herramienta del Sistema Nacional de Mejora Regulatoria. El objetivo de la iniciativa es garantizar que los beneficios de la regulación sean superiores a sus costos y que la selección normativa sea la mejor alternativa para determinado problema, sin desconsiderar el interés general (MÉXICO, 2018). En España, la denominada Ley de Procedimiento Administrativo Común de las Administraciones Públicas integra la AIR de forma implícita. De acuerdo con la ley, la norma regulatoria debe ser adecuada a los principios de la buena regulación, promoviendo el análisis económico de su impacto y evitando la institución de restricciones injustificadas o desproporcionados a la actividad económica (JEFATURA DEL ESTADO ESPAÑOL, 2015). En Chile, en abril de 2019, se publicó la Guía Chilena para la Buena Regulación, la cual incorpora el AIR para todos los proyectos de ley y normas infralegales, proponiendo

11 Es importante especificar que, a diferencia de Brasil, algunos países adoptan otra denominación de AIR. Por ejemplo, en Colombia, se llama Análisis de Impacto Normativo (AIN); en México, Declaración de Impacto Regulatorio; en el Reino Unido, Análisis de Impacto Regulatorio (RIA). 
una evaluación preliminar para determinar la necesidad, magnitud del impacto y la opción com mayor beneficio social (CHILE-MINISTERIO DE ECONOMÍA, 2019).

Sea en el contexto nacional o internacional, es evidente que la inclusión del Análisis de Impacto Regulatorio, así como las demás herramientas de mejora regulatoria, está progresivamente permeando los procesos decisorios de la regulación. Frente a eso, es natural que se pase a discusiones sobre aspectos específicos de su aplicación, como, por ejemplo, la utilización de recursos y medios tecnológicos. Siendo este específicamente el tema sobre el cual el presente trabajo pretende ir, en el próximo capítulo se abordará específicamente la utilización de la inteligencia artificial en el mecanismo del AIR.

\section{LA UTILIZACIÓN DE LA INTELIGENCIA ARTIFICIAL EN EL ÁMBITO DEL ANÁLISIS DE IMPACTO REGULATORIO (AIR): COMPATIBILIDAD, CONSIDERACIONES Y PRESUPUESTOS}

La inteligencia artificial (a partir de ahora denominada IA) es un término cuya definición no es unívoca, pero es posible destacar la concordancia en cuanto al menos a tres de sus características: (i) intencionalidad, en el sentido de que el sistema algorítmico no opera de modo pasivo y de relativa autónoma para ejercer tareas específicas; (ii) la inteligencia propiamente dicha, una vez que el sistema aprende en una secuencia semejante a la humana; (iii) y adaptabilidad, pues ostenta la capacidad cognitiva de efectuar ajustes a la medida que recolecta vastas informaciones (FREITAS, 2019 , p. 16). De este modo, al mismo tiempo en que la inteligencia artificial no se confunde con la automación, tan poco puede ser equiparada a la inteligencia humana, ya que está aquella que irá a condicionar la programación de aquella.

Entre las múltiples aplicaciones de la inteligencia artificial al Derecho que deben recibir especial atención está la asistencia digital para la toma de la decisión pública (FREITAS, 2019, p. 19). El tema de la utilización de la inteligencia artificial en el sistema jurídico ha sido pautado cada vez más a menudo, especialmente en el ámbito de la intervención regulatoria estatal. En este campo, la promesa es de prevenir las fallas del mercado (como, por ejemplo, las informaciones asimétricas, el abuso del poder dominante y las externalidades negativas) y las fallas de gobierno (como, por ejemplo, 
la omisión de prevención y precaución y el patrimonialismo), mediante incorporación de "valores elevados al nivel de algoritmos, en la cabal sujeción a la dignidad y al derecho al futuro" (FREITAS, 2019, p. 17-18). La promesa es tentadora, pues pretende sustituir un modelo de regulación errático y susceptible al patrimonialismo por un modelo óptimo, esto es, pretende promover la llamada "Smart regulation".

La incorporación de IA en el proceso regulatorio, en el ámbito del AIR, contribuye con la potencialización de la función, pues da asistencia para la toma de decisiones públicas. En ese sentido, su uso podría optimizar el flujo de datos e información que se encuentra a disposición del gestor público, pues pasaría a ser posible la resolución de cuestiones que anteriormente requerirían múltiples pasos, procedimientos y fases (CORVALÁN, 2017, p. 58). La nueva tecnología y el instrumento de mejora regulatoria son compatibles en la medida en que ambas figuras están orientadas, entre otras cosas, a la reducción de costos y promoción de análisis sistemáticos y fidedignos a la realidad. El resultado de la unión de ambas no será otro sino la calidad regulatoria mediante la priorización de esfuerzos y simplificación de la toma de decisión (OCDE, 2016, p. 122).

El AIR, más allá de ser una herramienta regulatoria que permite evaluar los probables beneficios, costos y efectos de la norma a ser editada, es un proceso auxiliar a la toma de decisiones regulatorias (OCDE, 2008). De este modo, la IA gana relevancia e importancia como el proceso lógico de formación del AIR. Con el apoyo de aquella, la recolección y organización de datos puede ser hecha de modo inteligente, conducida de acuerdo con el problema regulatorio que se pretende sanar. Más allá de eso, se puede programar la provisión de diversas alternativas de solución a ser dadas por el propio sistema, lo que apenas incrementa la tecnicidad de decisión regulatoria y aumenta su adherencia y conformidad con la realidad.

Un buen ejemplo práctico puede ser dado en el ámbito de la regulación del transporte urbano. Suponiendo que se observa empíricamente que el sistema de transporte público de una ciudad ya no es más efectivo, el ente regulador debe investigar las causas, listar las posibles soluciones y tener criterios claros para optar por una de ellas. Por tanto, es necesario sintetizar una realidad que puede ser aprendida de numerosos datos. En el caso hipotético, la IA podría ser utilizada para identificar varios aspectos del flujo de pasajeros: la cantidad, el punto de partida y el punto de llegada, horarios de pico, líneas y carros llenos, entre otros. Las soluciones podrían ser varias: alteración de ruta, inclusión de nuevas líneas o carros, estímulo 
a la locomoción por medios alternativos (como bicicletas y patinetas) en determinadas localizaciones, entre otros. Esa tarea sería tortuosa, costosa y demorada si es realizada a penas por el gestor público, sino imposible. Con el apoyo de la IA en el proceso de AIR, entre tanto, sería rápida y precisa.

En otros países es posible observar de forma clara la conjugación de la inteligencia artificial en el proceso regulatorio. En Australia, la adaptación a las transformaciones tecnológicas hace parte de los principios de la regulación, cuya aplicación práctica es orientada para la identificación de datos e instrumentos que fortalecen la mejor decisión de los agentes públicos (AUSTRALIA- COMISSIONER FOR BETTER REGULATION, 2016), con la finalidad de mejorar los procesos regulatorios en términos de evaluación y revisión de costos y beneficios (AUSTRALIA, 2007). La Comisión Europea, por su vez, dispone que la inteligencia artificial, con apoyo de big data, ${ }^{12}$ crea nuevas posibilidades para el análisis de circunstancias sociales y económicas (COMISIÓN EUROPEA, 2015, p. 17). En el país de Moldavia, el gobierno elaboró una guía metodológica para la evaluación del impacto de la regulación de políticas públicas. En el documento, se consignó que este presupone la utilización de medios tecnológicos que viabilicen la obtención de referencias y consultorías durante la evaluación de impacto (sugerencia de soluciones y simulación de escenarios, por ejemplo). Esto es posible apenas por intermedio de la inteligencia artificial (MOLDOVIA, 2009, p. 57).

De modo general, la introducción de herramientas tecnológicas en el proceso de identificación de problemas, alternativas y sus impactos, es una transformación que demanda esfuerzos (MENENGUIN \& SILVA, 2017), pero que converge para la consolidación de un Gobierno y una Administración Pública digital (STRINGHINI, 2018), modelo en el cual la verificación de aciertos y desaciertos es más asertiva (BRASIL-MINISTÉRIO DO PLANEJAMENTO, 2019).

No obstante los numerosos beneficios derivados de la IA en el proceso regulatorio en la fase del AIR, el fenómeno debe ser analizado como parsimonia a la luz del ordenamiento jurídico, especialmente en relación a los derechos y garantías fundamentales. Por tanto, más allá de proponer la referida práctica, en el presente trabajo también son listados los presupuestos para tanto. Y ellos son (i) en relación a la extensión de la posibilidad de utilización de inteligencia artificial; (ii) en relación al modo de implementación de la inteligencia artificial.

12 Término que designa grandes conjuntos de datos que pueden ser processados y aarmonizados. 
En cuanto al primer aspecto, la IA debe ser implementada sin que se sustituya la decisión final por el propio agente público. Por cierto la función de la práctica es viabilizar el apoyo digital mediante el auxilio en la identificación de problemas y sugerencias de solución, pero la decisión debe ser siempre de aquel que detiene la competencia para eso. ${ }^{13}$ De este modo, el gestor no estará vinculado a aplicar conclusiones provistas por la IA, pero tan solamente las utiliza en la motivación de su decisión. Este es un límite ético importante por lo menos en dos razones: para evitarse la eventual "tirania digital", para resguardar el inevitable componente subjetivo que integra la discrecionalidad (SANTOFIMIO, 2019, p. 17-42); y para evitar la eliminación del componente humano de una función cuyo fin último es justamente el bienestar de los ciudadanos.

En segundo lugar, en cuanto al modo de implementación de la inteligencia artificial, Juarez Freitas ya destacó pioneramente que se debe garantizar el acceso a la secuencia de los pasos lógicos de la decisión algorítmica. La exigencia se justifica porque mucho antes de que el análisis de datos por inteligencia artificial aparente ser un proceso neutro, ese no es. Detrás de la inteligencia artificial está la programación hecha por un ser humano, que como tal puede ser tendenciosa e viciada. Un ejemplo puede ayudar a compreender la importancia de ese presupuesto: caso en que una persona racista promueva el análisis de regulación del servicio público de agua, ella podría indebidamente crear algoritmos tendenciosos a indicar que regiones más habitadas por negros no requiere de mejorías en el servicio.

Finalmente, hay todavía un segundo requisito en relación al modo de la implementación de la inteligencia artificial. Como fue visto, la inteligencia artificial es desarrollada a partir de una cantidad significativa de datos, pues es apenas a partir de estos que se puede identificar patrones y dar secuencia a pasos lógicos. En términos de regulación, los datos serán necesariamente de los particulares: empresas reguladas, usuarios de servicios públicos y consumidores. La situación atrae la incidencia de las normas de la Ley General de Protección de Datos, en especial a las disposiciones sobre el tratamiento de datos personales por parte del Poder Público. Considerando el alcance y extensión del presente trabajo, no se realizará un análisis minucioso de cuáles son las referidas disposiciones y de cuál modo, en la prática, ellas deberán ser observadas. Por ahora cumple resaltar que la

13 Como André Luiz Freire señaló muy bien, el alcance de las actividades públicas está guiado por el principio de competencia, según el cual el Estado no puede ser excusado de proporcionarle algo confiado. (FREIRE, 2014, p. 246). 
Administración Pública, al desempeñar la función reguladora con apoyo de la Inteligencia Artificial, no está exenta de respetar los derechos inherentes a las personas que opera.

\section{CONSIDERACIONES FINALES}

El paradigma moderno según el cual la Administración sólo podría actuar de acuerdo con la estricta subsunción a la ley fue superado. Actualmente se reconoce la prevalencia del paradigma neoconstitucional, caracterizado por Constituciones con un extenso catálogo de derechos fundamentales, bien como valores y principios que trasladan la noción de legalidad para la noción de juridicidad. Esa transformación de paradigma impacta directamente en la Administración Pública, que pasa a administrar los intereses públicos que constitucionalmente le fueron atribuidos en vez de actuar de modo legalista. Surge entonces, el denominado Derecho Administrativo postmoderno.

El Derecho Administrativo postmoderno repercute en toda la actividad administrativa, especialmente en la actividad regulatoria independiente. Las leyes que instituyen sistemas de regulación tienen menor densidad normativa, lo que abre espacio para una mayor discrecionalidad de la Administración Pública. Ese factor aumenta la complejidad del análisis de legalidad de los actos, teniendo en cuenta que en vez de un análisis meramente legalista, son necesarias evaluaciones más complejas sobre proporcionalidad, razonabilidad e imparcialidad.

El objetivo de la regulación es garantizar el mejor funcionamiento y desarrollo posible del mercado regulatorio, considerando los intereses empresariales, pero también de los usuarios de los servicios y la sociedad como un todo, bien como del propio Estado. Siendo así, la finalidad de garantizar el desarrollo está inmerso en el propio concepto de regulación, y ese desarrollo debe ser comprendido desde el punto de vista económico, ambiental, social y político, considerando el aspecto de la sostenibilidad.

Para que la función regulatoria alcance esa finalidad, es imprescindible que la decisión regulatoria sea técnica y sea tomada con fundamento en la mayor cantidad posible de informaciones que evidencie la realidad, para que pondere forma adecuada de todos los intereses involucrados. Por tanto, es de extrema relevancia la discusión sobre los instrumentos que puedan incrementar la referida tecnicidad, entre ellos, la incorporación del Análisis de Impacto Regulatorio con Inteligencia Artificial con apoyo de la decisión regulatoria. 
La regulación inteligente es un concepto que pasó por transformaciones, se puede definir como una política cuya finalidad no es apenas el incremento de la participación de los interesados, sino también el aumento de la tecnicidad de las decisiones $\mathrm{y}$, consecuentemente, de la maximización de su efectividad y eficiencia. Son diversos los instrumentos para llevar a cabo la regulación inteligente, entre ellos el Análisis de Impacto Regulatorio (AIR).

El AIR consiste en un proceso de análisis de los impactos de la norma regulatoria que propicia la medición del costo-beneficio de la norma a ser editada, tanto desde el punto de vista económico cuanto desde el punto de vista social, ambiental, político y cultural. La utilización de la herramienta aumenta la efectividad de la regulación, aumenta la participación de los interesados, aumenta la tecnicidad de la decisión y establece parámetros más claros para su control. Considerando sus beneficios, la herramienta fue progresivamente incorporada al ordenamiento jurídico brasilero y en los demás países.

El aumento en la utilización de la herramienta conduce a debates específicos, tales como la utilización de la inteligencia artificial (IA) para la asistencia digital de la toma de decisión pública. La utilización de la IA en el AIR trae consigo la promesa de prevenir fallas de mercado y fallas de gobierno mediante el análisis de todos los datos e informaciones que se encuentran a disposición del gestor público. Siendo así, la nueva tecnología y el instrumento de mejora regulatoria son compatibles en la medida en que ambas figuras están orientadas, entre otras cosas, a la reducción de costo y a la promoción de análisis sistemáticos y fidedignos a la realidad.

A pesar de los beneficios que pueden derivarse, la utilización de la IA en el proceso regulatorio debe ser analizada con cautela, a la luz del ordenamiento jurídico. Por lo tanto, el presente trabajo defendió que tal práctica debe efectivarse de acuerdo con los siguientes presupuestos. Con relación a la extensión de la posibilidad de la utilización de la inteligencia artificial, se debe alertar y hacer seguimiento al permitir que la IA sustituya la decisión del agente público. Finalmente, de acuerdo al modo de implementación de la inteligencia artificial. Se le debe dar publicidad a la secuencia de pasos lógicos de la decisión algorítmica. Así como se debe orientar las disposiciones de la ley General de Protección de Datos. 


\section{REFERENCIAS}

STRINGHINI, A. Administración pública inteligente: novedades al ecosistema normativo digital de la República Argentina. Revista Eurolatinoamericana de Derecho Administrativo, v. 5, n. 2, p. 199-216, 28 dez. 2018.

ANVISA - AGÊNCIA NACIONAL DE VIGILÂNCIA SANITÁRIA. Análise de Impacto Regulatório - Processo 25351365875200953. Brasília, DF, 2018. Disponible en: http://portal.anvisa.gov.br/analise-de-impacto-regulatorio?p_p_id=110. Acceso: 19 de septiembre. 2019.

ARAGÃO, A. S. O poder normativo das agências reguladoras independentes e o Estado democrático de Direito. Revista de Informação Legislativa, Brasília, DF, año 37, n. 148, p. 273-299, oct./dic. 2000.

AUSTRALIA. Best practice regulation handbook, 2007. Disponible en: http://regulationbodyofknowledge.org/wp-content/uploads/2013/03/AustralianGovernment_Best_Practice_Regulation.pdf. Acceso: 19 de septiembre. 2019.

AUSTRALIA. Commissioner for Better Regulation. Victorian guide to regulation: a handbook for policy-makers in Victoria (Australia), 2016. Disponible en: http://www.betterregulation.vic.gov.au/files/98181269905c-4893-bff3-a6bb009df93c/Victorian-Guide-to-Regulation-PDF-final. pdf. Acceso: 19 de septiembre. 2019.

BACELLAR FILHO, R. F.; PIVETTA, S. L. O regime jurídico do processo administrativo na Lei n. 9.784/99. A\&C - Revista de Direito Administrativo \& Constitucional, Belo Horizonte, año 14, n. 58, p. 107-135, oct./ dic. 2014.

BETANCOR, A. Mejorar la regulación: una guía de razones y medios. Madrid: Marcial Pons, 2009.

BLANCHET, L. A.; BUBNIAK, P. L. T. Análise de Impacto Regulatório: uma ferramenta e um procedimento para a melhoria da regulação. Revista de Ciências Jurídicas, Fortaleza, v. 22, n.3, p. 1-15, sep./dic. 2017.

BRASIL. Casa Civil da Presidência da República. Inventário AIR - Visão Geral da Análise de Impacto Regulatório nas Agências Reguladoras Federais. Brasília, DF 20 jun. 2018. 
BRASIL. Congresso Nacional. Projeto de Lei n. 1.539/2015. Disponible en: https://www.camara.leg.br/proposicoesWeb/prop_mostrarintegra;jsessionid=0E6A4D328962E24E5E9213B11A5CBC21.proposicoesWeb2?codteor $=1334093 \&$ filename $=P L+1539 / 2015$. Acceso: 19 de septiembre. 2019.

BRASIL. Ministério do Planejamento, Desenvolvimento e Gestão. Estratégia de governança digital - EGD. Brasília, DF, 2019. Disponible en: https://www.governodigital.gov.br/EGD/documentos/revisao-da-estrategia-de-governanca-digital-2016-2019.pdf. Acceso: 19 de septiembre. 2019.

BRASIL. Subchefia de Análise e Acompanhamento de Políticas Governamentais. Diretrizes gerais e guia orientativo para elaboração de Análise de Impacto Regulatório - AIR /. Brasília, DF: Presidência da República, 2018. Disponible en: http:/www.casacivil.gov.br/governanca/regulacao/ apresentacao-regulacao-pasta/acesse-aqui/comite-interministerial-de-governanca-aprova-as-diretrizes-gerais-e-roteiro-analitico-sugerido-para-analise-de-impacto-regulatorio-diretrizes-air-e-o-guia-orientativo-para-elaboracao-de-analise-de-impacto-regulatorio-guia-air/diretrizes_guia air_cig_11junho2018.pdf. Acceso: 19 de septiembre. 2019.

CARBONELL, M. (Org.). Neoconstitucionalismo(s). Madrid: Trotta, 2003.

CASTRO, C. M. Some aspects of implementing Regulatory Impact Analysis in Brazil. Revista de Administração Pública, Rio de Janeiro, v. 48, n. 2 , p.323-342, abr. 2014.

CHILE. Ministerio de Economía, Fomento y Turismo. Guía chilena para una buena regulación. Santiago, 2019. Disponible en: https://open.economia.cl/wp-content/uploads/2019/04/Guia-Chilena-2019_25abril.pdf. Acceso: 10 de octubre. 2019.

COLOMBIA. Departamento Nacional De Planeación (DNP). Colombia da los primeros pasos hacia una regulación inteligente basada en la participación de los ciudadanos. 2018. Disponible en: https://www.dnp. gov.co/Paginas/Colombia-da-los-primeros-pasos-hacia-una-regulaci\%C3\%B3n-inteligente-basada-en-la-participaci\%C3\%B3n-de-los-ciudadanos.aspx. Acceso: 9 de mayo. 2018. 
CONSEJO EUROPEO. Comunicación n. 543, de 08 de outubro de 2010. Comunicación de La Comisión al Parlamento Europeo, al Consejo, al Comité Económico y Social Europeo y al Comité de las Regiones: Documento para trabajo 2011. Bruselas: Consejo Europeo, 8 oct. 2010.

CORVALÁN, J. G. Administración Pública digital e inteligente: transformaciones en la era de la inteligencia artificial. Revista de Direito Econômico e Socioambiental, Curitiba, v. 8, n. 2, p. 26-66, mayo/ago. 2017.

EUROPEAN COMMISSION. Better Regulation “Toolbox", 2015, p. 17. Disponible en: https://ec.europa.eu/info/law/law-making-process/planning-and-proposing-law/better-regulation-why-and-how/better-regulation-guidelines-and-toolbox_en. Acceso: 19 de septiembre. 2019.

FREIRE, A. L. O Regime de direito público na prestação de serviços públicos por pessoas privadas. São Paulo: Malheiros, 2014.

FREITAS, J. Direito Administrativo e inteligência artificial. Interesse Público - IP, Belo Horizonte, año 21, n. 114, p. 15-29, mar./abr. 2019.

FREITAS, J. Sustentabilidade: direito ao futuro. Belo Horizonte: Fórum, 2011.

GABARDO, E. Interesse público e subsidiariedade: o Estado e a sociedade civil para além do bem e do mal. Belo Horizonte: Fórum, 2009.

GUERRA, S. Discricionariedade, regulação e reflexividade: uma nova teoria sobre as escolhas administrativas. 5. ed. Belo Horizonte: Fórum, 2018 .

GUNNINGHAM, N.; SINCLAIR, D. Smart regulation. In: DRAHOS, P. Regulatory theory: foundations and applications. Camberra: Australia National University, 2017. p. 133-149. Disponible en: https://press-files.anu. edu.au/downloads/press/n2304/pdf/book.pdf. Acceso: 10 de abril. 2019.

HACHEM, D. W. A noção constitucional de desenvolvimento para além do viés econômico: reflexos sobre algumas tendências do Direito Público brasileiro. A\&C-Revista de Direito Administrativo \& Constitucional, Belo Horizonte, año 13, n. 53, p. 133-168, jul./set. 2013.

JEFATURA DEL ESTADO ESPAÑOL. Lei n. 39, de 1 de octubre de 2015. Del Procedimiento Administrativo Común de Las Administraciones Públicas. Madrid, 2015. Disponible en: https://www.boe.es/buscar/act.php?id=BOE-A-. Acceso: 10 de octubre. 2019. 
MASSIMINO, L. F. La intervención estatal, la regulación económica y el poder de policía. Revista de Direito Econômico e Socioambiental, Curitiba, v. 6, n. 677, p. 36-63, 2015.

MEDAUAR, O. A processualidade no Direito Administrativo. 2. ed. São Paulo: Revista dos Tribunais, 2008.

MELLO, C. A. B. Serviço público e concessão de serviço público. São Paulo: Malheiros, 2017.

MENDONÇA, J. V. S. Art. 21 da LINDB - indicando consequências e regularizando atos e negócios. Revista de Direito Administrativo, Rio de Janeiro, p. 43-61, nov. 2018.

MENEGUIN, F. B.; BIJOS, P. R. S. Avaliação de impacto regulatório: como melhorar a qualidade das normas. Brasília, DF: Senado Federal, 2016. Disponible en: https://www2.senado.leg.br/bdsf/bitstream/handle/ $\mathrm{id} / 519196 /$ OED0024.pdf? sequence=1\&isAllowed=y. Acceso: 19 de septiembre. 2019.

MENEGUIN, F.; SILVA, R. S. (Orgs.). Avaliação de Impacto legislativo: cenários e perspectivas para sua aplicação, Brasília, DF: Senado Federal, 2017. Disponible en: https://www2.senado.leg.br/bdsf/bitstream/handle/ $\mathrm{id} / 519163 /$ TD193.pdf? sequence=1\&isAllowed=y. Acceso: 19 de septiembre. 2019 .

MÉXICO. Congreso General de la Unión de los Estados Unidos Mexicanos. 18 de maio de 2018. La Ley General de Mejora Regulatoria. Ciudad de México, 2018. Disponible en: http://dof.gob.mx/nota_detalle.php?codigo $=5523172 \&$ fecha $=18 / 05 / 2018$. Acceso: 17 de septiembre. 2019

MOLDOVIA. Methodological guide on ex-ante assessment of the impact of Public policies, 2009. Disponible en: https://www.legislationline.org/ documents/id/17155. Acceso: 19 de septiembre. 2019.

MORAN, M. Review article: understanding the Regulatory State. British Journal of Political Science, v. 2, n. 2, p. 391-413, mar. 2002.

MOREIRA NETO, D. F. (Coord.). Uma avaliação das tendências contemporâneas do Direito Administrativo. Rio de Janeiro: Renovar, 2003.

MOREIRA NETO, D. F. Mito e realidade do serviço público. Revista de Direito da Procuradoria Geral do Estado do Rio de Janeiro - RDPGE, Rio de Janeiro, v. 53, p. 140-141, 2000. 
MOREIRA NETO, D. F. O direito administrativo do século XXI: um instrumento de realização da democracia substantiva. A\&C - Revista de Direito Administrativo \& Constitucional, Belo Horizonte, año 11, n. 45, p. 13-37, jul./sep. 2011.

MOREIRA NETO, D. F. Quatro paradigmas do direito administrativo pós-moderno: legitimidade, finalidade, eficiência, resultados. Belo Horizonte: Fórum, 2008.

MORENO, L., GALLO, W. De la simplificación administrativa a la calidad regulatoria. Revista del Círculo de Derecho Administrativo, v. 17, n. 17, p. 246-273, 2019. Disponible en: https://www.academia.edu/40551524/Simplifacio_n_Administrativa_Ferney_y_Gallo_-_RDA_17_1_Acceso: 19 de septiembre. 2019.

OCDE - ORGANIZAÇÃO PARA A COOPERAÇÃO E DESENVOLVIMENTO ECONÔMICO. Panorama de las Administraciones Públicas: América Latina y el Caribe 2017. Paris: Éditions OCDE, 2016.

OCDE - ORGANIZAÇÃO PARA A COOPERAÇÃO E DESENVOLVIMENTO ECONÔMICO. Building an institutional framework for regulatory impact analysis. Version 1.1 Regulatory Policy Division Directorate for Public Governance and territorial Development. Paris, 2008. Disponible en: http://www.oecd.org/gov/regulatory-policy/40984990.pdf. Acceso: 12 de diciembre. 2015.

RAGAZZO, C. Coordenação efetiva e sistematização: novas tendências da melhora da qualidade regulatória no Brasil. Revista Estudos Institucionais, Rio de Janeiro, v. 4, n. 2, p. 513-536, jul./dic. 2018.

RODRÍGUEZ-ARANA MUÑOZ, J. El derecho fundamental a la buena administracion en la constitución española y en la Unión Europea. Revista Eurolatinoamericana de Derecho Administrativo, v. 1, n. 2, p. 73-93, dic. 2014.

SANTOFIMIO GAMBOA, J. O. Tecnología y inteligencia artificial: incidencias en el derecho aplicable a la administración pública: dos ideas en torno a su futuro inmediato de frente al cumplimiento eficaz de los propósitos y finalidades de la función pública administrativa. In: ZEGARA VALDIVIA, D. La proyección del Derecho Administrativo Peruano: estudios por el Centenario de la Facultad de Derecho de la PUCP. Lima: Palestra, 2019. p. 17-42. 
SCHIER, A. Serviço público: garantia fundamental e cláusula de proibição de retrocesso social. Curitiba: Íthala, 2016.

SCHIER, A. Fomento: administração pública, direitos fundamentais e desenvolvimento. Curitiba: Íthala, 2019.

SCHIRATO, V. R. A deterioração do sistema regulatório brasileiro. Revista de Direito Público da Economia - RDPE, Belo Horizonte, año 11, n. 44, p. 249-274, oct./dic. 2013.

SCHIRATO, V. R. Livre iniciativa nos serviços públicos. Belo Horizonte: Fórum, 2012.

SILVA, L. N. O processo de globalização e a instabilidade dos modelos econômicos de Estado. Revista de Informação Legislativa, Brasília, DF, año 41, n. 163, p. 331-346, jul./set. 2004.

SUSTEIN, C. Costs, benefits and regulation post-Trump. Bloomerang The Company, 1 ago. 2019. Disponible en: https://www.bloomberg.com/opinion/articles/2019-08-01/cost-benefit-analysis-regulation-after-trump. Acceso: 19 de septiembre. 2019.

TOFFELSON, C, et.al. Setting the standard: certification, governance and the Forest Stewardship Council: Vancouver: UBC Pres, 2008.

Artículo recibido el: 15/04/2020.

Artículo aceptado el: 20/10/2020.

\section{Cómo citar este artículo (ABNT):}

GALLO APONTE, W. I.; LÓPEZ VALLE, V. C.; FÁCIO, R. N. La utilización de inteligencia artificial en la actividad regulatoria: una propuesta en favor del desarrollo nacional sostenibl. Veredas do Direito, Belo Horizonte, v. 17, n. 39, p. 123-146, sep./dic. 2020. Disponible en: http://www. domhelder.edu.br/revista/index.php/veredas/article/view/1830. Acceso: día del mes. año. 